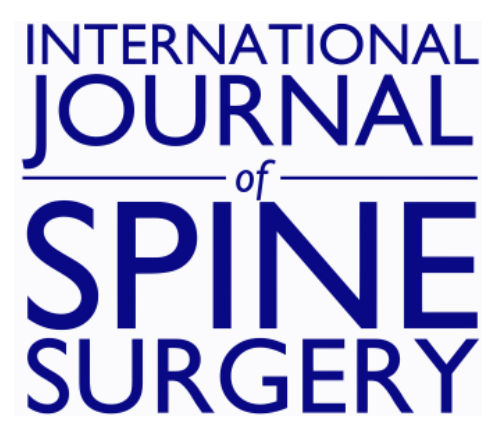

\title{
T1 Slope Minus Cervical Lordosis (TS-CL), the Cervical Answer to PI-LL, Defines Cervical Sagittal Deformity in Patients Undergoing Thoracolumbar Osteotomy
}

\begin{abstract}
THEMISTOCLES PROTOPSALTIS, JAMIE TERRAN, ALEX SOROCEANU, MICHAEL J. MOSES, NICOLAS BRONSARD, JUSTIN SMITH, ERIC KLINEBERG, GREGORY MUNDIS, HAN JO KIM, RICHARD HOSTIN, ROBERT Hart, CHRISTOPHER SHAFFREY, SHAY BESS, CHRISTOPHER AMES, FRANK SCHWAB, VIRGINIE LAFAGE and INTERNATIONAL SPINE STUDY GROUP
\end{abstract}

Int J Spine Surg 2018, 12 (3) 362-370

doi: https://doi.org/10.14444/5042

http://ijssurgery.com/content/12/3/362

This information is current as of April 26, 2023.

Email Alerts Receive free email-alerts when new articles cite this article. Sign up at: http://ijssurgery.com/alerts 


\title{
T1 Slope Minus Cervical Lordosis (TS-CL), the Cervical Answer to PI-LL, Defines Cervical Sagittal Deformity in Patients Undergoing Thoracolumbar Osteotomy
}

\author{
THEMISTOCLES PROTOPSALTIS, ${ }^{1}$ JAMIE TERRAN, ${ }^{1}$ ALEX SOROCEANU,${ }^{2}$ MICHAEL J. MOSES, ${ }^{1}$ \\ NICOLAS BRONSARD,${ }^{3}$ JUSTIN SMITH,${ }^{4}$ ERIC KLINEBERG,${ }^{5}$ GREGORY MUNDIS, ${ }^{6}$ HAN JO KIM, ${ }^{7}$ \\ RICHARD HOSTIN,${ }^{8}$ ROBERT Hart,${ }^{9}$ CHRISTOPHER SHAFFREY, ${ }^{4}$ SHAY BESS, ${ }^{10}$ CHRISTOPHER \\ AMES, ${ }^{11}$ FRANK SCHWAB, ${ }^{7}$ VIRGINIE LAFAGE, ${ }^{7}$ INTERNATIONAL SPINE STUDY GROUP \\ ${ }^{1}$ New York University School of Medicine, Department of Orthopedic Surgery, New York, New York, ${ }^{2}$ Department of Orthopaedic Surgery, University of Calgary, \\ Calgary, Alberta, Canada, ${ }^{3}$ Department of Orthopaedic, Trauma, and Spine Surgery, Institut Universitaire de l'appareil Locomoteur et du Sport, Hôpital Pasteur \\ 2, Centre Hospitalier Universaire de Nice, Nice, France, ${ }^{4}$ University of Virginia School of Medicine, Department of Neurosurgery, Charlottesville, Virginia, \\ ${ }^{5}$ University of California Davis, Department of Orthopedic Surgery, Sacramento, California, ${ }^{6}$ San Diego Center for Spinal Disorders, La Jolla, California, \\ ${ }^{7}$ Hospital for Special Surgery, Department of Orthopedic Surgery, New York, New York, ${ }^{8}$ Baylor Scoliosis Center, Plano, Texas, ${ }^{9}$ University of Oregon Health \\ Sciences Center, Department of Orthopedic Surgery, Portland, Oregon, ${ }^{10}$ Rocky Mountain Hospital for Children, Presbyterian/St Luke's Medical Center, Denver, \\ Colorado, "'University of California San Francisco, Department of Neurosurgery, San Francisco, California
}

\begin{abstract}
Background: Cervical kyphosis and C2-C7 plumb line (CPL) are established descriptors of cervical sagittal deformity (CSD). Reciprocal changes in these parameters have been demonstrated in thoracolumbar deformity correction. The purpose of this study was to investigate the development of CSD, using T1 slope minus cervical lordosis (TS-CL) to define CSD and to correlate TS-CL and a novel global sagittal parameter, cervical-thoracic pelvic angle (CTPA), with CPL.

Methods: A multicenter, retrospective analysis of patients with thoracolumbar deformity undergoing threecolumn osteotomy was performed. Preoperative and postoperative cervical parameters were investigated. Linear regression for postoperative values resulted in a CPL of $4 \mathrm{~cm}$ corresponding to a TS-CL threshold of $17^{\circ}$. Patients were classified based on postoperative TS-CL into uncompensated (TS-CL $>17^{\circ}$ ) or compensated cohorts $\left(\mathrm{TS}-\mathrm{CL}<17^{\circ}\right.$ ); the two were compared using an unpaired $t$ test. Logistic regression modeling was used to determine predictors of postoperative CSD.

Results: A total of 223 patients with thoracolumbar deformity (mean age, 57.56 years) were identified. CTPA correlated with CPL (preoperative $r=.85$, postoperative $r=.69$ ). TS-CL correlated with CTPA (preoperative $r=.52$, postoperative $r=.37$ ) and CPL (preoperative $r=.52$; postoperative $r=.37$ ). CSD had greater preoperative CPL $(P<$ $.001)$ and CTPA $(P<.001)$. The compensated cohort had a decrease in TS-CL (from 10.2 to 8.0) with sagittal vertical axis (SVA) correction, whereas the uncompensated had an increase in TS-CL (from 22.3 to 26.8) with all $P<.001$. Reciprocal change was demonstrated in the compensated group given that CL decreased with SVA correction $(r=.39)$, but there was no such correlation in the uncompensated. Positive predictors of postoperative CSD included baseline TS$\mathrm{CL}>17^{\circ}(P=.007)$, longer fusion $(P=.033)$, and baseline CTPA $(P=.029)$.

Conclusions: TS-CL and CTPA correlated significantly with established sagittal balance measures. Whereas reciprocal change in cervical and thoracolumbar alignment was demonstrated in the compensated cohort, the uncompensated population had progression of their cervical deformities after three-column osteotomy.

Clinical Relevance: The balance between TS-CL mirrors the relationship between pelvic incidence minus lumbar lordosis in defining deformities of their respective spinal regions.

Cervical Spine

Keywords: cervical kyphosis, thoracolumbar deformity, alignment, TS-CL, CPL
\end{abstract}

\section{INTRODUCTION}

There is an interplay of regional spinal alignment in the economy of standing posture that has been referred to as the "chain of correlation." 1,2 In order to achieve harmonious spinopelvic alignment, large pelvic incidence requires larger lumbar lordosis, larger thoracic kyphosis, and greater cervical lordosis, which is also important in the maintenance of horizontal gaze. ${ }^{3}$ The use of modern spinal deformity surgical techniques such as the pedicle subtraction osteotomy has enabled large global deformity 
corrections across fused segments. However, there has been increasing recognition that reciprocal changes in alignment occur in adjacent regions of the spine. Reciprocal thoracic hyperkyphosis occurring across unfused segments following lumbar osteotomy has also been demonstrated. ${ }^{4,5}$ Conversely, thoracic osteotomies and limited fusions for the correction of thoracic kyphosis have been shown to decrease lumbar lordosis. ${ }^{4}$ Smith et $\mathrm{al}^{6}$ exhibited that patients with thoracolumbar sagittal imbalance with sagittal vertical axis (SVA) $>5 \mathrm{~cm}$ had reciprocal cervical hyperlordosis that resolved following correction of the underlying thoracolumbar deformity. A recent study revealed that among patients with thoracolumbar deformities, there was a prevalence of cervical deformities such as increased C2-C7 plumb line (CPL) and cervical kyphosis. ${ }^{6} \mathrm{~A}$ CPL or cervical offset of greater than $4 \mathrm{~cm}$ has been shown to be associated with greater disability by validated outcome measures. ${ }^{7}$ However, cervical offset may not be the best descriptor of primary cervical deformity in the setting of an underlying thoracolumbar deformity. Smith et $\mathrm{al}^{8}$ demonstrated that the correction of the thoracolumbar sagittal malalignment also led to improved cervical offset with a decrease in the CPL. Thus, deformity in the other portions of the spine can insidiously affect the cervical alignment and the respective measurements of cervical deformity.

With greater recognition of the influence of global spinal alignment on cervical deformity, several authors have shown that cervical kyphosis can be physiologic, depending on the underlying global spinopelvic alignment. ${ }^{9,10}$ Pelvic incidence and lumbar lordosis are two measures that have been well established in playing a role in balancing global spinal alignment. ${ }^{11-13}$ The pelvic incidence minus lumbar lordosis (PI-LL) parameter correlates with health-related quality of life measures in patients with thoracolumbar deformity. ${ }^{11-13}$ In fact, lumbar lordosis is more highly correlated to sacral slope (SS) than pelvic incidence in asymptomatic patients. $^{2,14}$ An analogous cervical correlate is the balance between cervical lordosis (CL) and T1 slope (TS). A direct relationship between these two parameters has been inferred, but the use of TS and $\mathrm{CL}$ as a descriptor of primary cervical deformity has not been well established. . $^{815}$

The purpose of this study was to investigate the use of TS-CL as a marker of cervical sagittal deformity (CSD) and to evaluate the development of CSD following thoracolumbar three-column osteotomy. We expect TS-CL to correlate with the variety of existing descriptive measures of CSD and to more reliably identify primary CSD especially in the setting of concurrent thoracolumbar deformity.

\section{MATERIALS AND METHODS}

\section{Patient Selection}

This study was a multicenter retrospective review of patients who underwent three-column osteotomies. Data were collected at 8 sites across the United States with institutional review board approval. Inclusion criteria for this study were patients with age $>18$ years, full standing scoliosis radiographs including the cervical spine to the pelvis at the preoperative and 1-year postoperative time points, and thoracic or lumbar three-column osteotomy (Schwab grade 3 or 4 ). ${ }^{16}$

\section{Data Collection}

Demographic and perioperative data were collected at each site. Office charts were reviewed for demographic variables including, age, height, weight, sex, and history of prior surgery. Preoperative and postoperative radiographs were included in the database. Surgical and intraoperative measures were collected based upon review of operative notes and anesthesia reporting. All radiographs included in the database had a minimum of $\mathrm{C} 7$ pelvis; however, only those also including the cervical spine were used in this analysis.

\section{Radiographic Parameters}

Preoperative and 1-year postoperative, sagittal cervical, thoracolumbar, and spinopelvic parameters were measured using Spineview software (Laboratory of Biomechanics, ENSAM ParisTech, Paris, France), ${ }^{17,18}$ a validated and computer-based tool that enables quantitative measures of the spine and pelvis. In addition, preoperative thoracolumbar deformity severity was quantified using the Scoliosis Research Society-Schwab Classification of adult spinal deformity. ${ }^{19}$

- Cervical radiographic parameters (Figure 1) included $\mathrm{C} 2$ slope (C2S), CPL, C2-C7 lordosis (CL), T1 slope (TS), and T1 slope minus cervical lordosis (TS-CL). 




Figure 1. Cervical radiographic parameters measured including the cervical Iordosis (CL), the $\mathrm{C} 2$ slope, the cervical plumb line $(\mathrm{CPL})$, and the $\mathrm{T} 1$ slope.

- Thoracolumbar and spinopelvic radiographic parameters included T2-T12 thoracic kyphosis (TK_T2), T4-T12 thoracic kyphosis (TK_T4), L1-S1 lumbar lordosis (LL), pelvic incidence (PI), Pelvic tilt, SS, and PI-LL.

- Global radiographic parameters (Figure 2) included SVA, cervico-thoracic pelvic angle (CTPA), C2 pelvic angle, and T1 pelvic angle (TPA).

\section{Statistical Analysis}

Analysis of total population radiographic and demographic parameters was conducted. Correlation of existing and novel radiographic parameters was reviewed. Because global sagittal correction is known to affect cervical sagittal malalignment, postoperative values were used in linear regression analysis to determine the threshold of TS-CL corresponding to a CPL of $4 \mathrm{~cm}$. This cervical alignment threshold was then used to classify patients into uncompensated (TS-CL above threshold) and compensated (TS-CL below threshold) groups (Figures 3 and 4). Radiographic parameters between groups were compared using unpaired $t$ test analysis. Radiographic correlations were reviewed within CSD and Reciprocal Sagittal Alignment groups using Pearson correlations. Level of significance was set to .05 .



Figure 2. Global radiographic parameters measured including the cervicothoracic pelvic angle (CTPA), the $\mathrm{C} 2$ pelvic angle (CPA), and the T1 pelvic angle (TPA). CTPA is a global angular measure of cervical sagittal alignment and a correlate of the $\mathrm{C} 2-\mathrm{C} 7$ plumb line. T1 pelvic angle is a measure of global sagittal alignment and a correlate of the $\mathrm{C} 7$ sagittal vertical axis. CPA is the angle of a line from $\mathrm{C} 2$ centroid to the femoral heads $(\mathrm{FH})$ and a line from the $\mathrm{FH}$ to the middle of the S1 endplate. T1 pelvic angle is the angle of a line from the center of $\mathrm{T} 1$ to the $\mathrm{FH}$ and a line from the $\mathrm{FH}$ to the center of the S1 endplate. CTPA is the angle of a line from $\mathrm{C} 2$ centroid to the $\mathrm{FH}$ and a line from the $\mathrm{FH}$ to the center of T1. CTPA is the result of subtracting T1 pelvic angle from CPA.

Logistic regression modeling was used to determine predictors of postoperative CSD (TS-CL above threshold). Potential predictors were identified using univariate analysis and expert opinion. The model was built using a combination of backward elimination and bootstrap selection. Our final model had 20 events per variable. Model fit was assessed using the Hosmer-Lemeshow test and the $\mathrm{C}$ statistic/receiver operating characteristic curve. Internal validation of the model was performed using 10-fold cross-validation. 

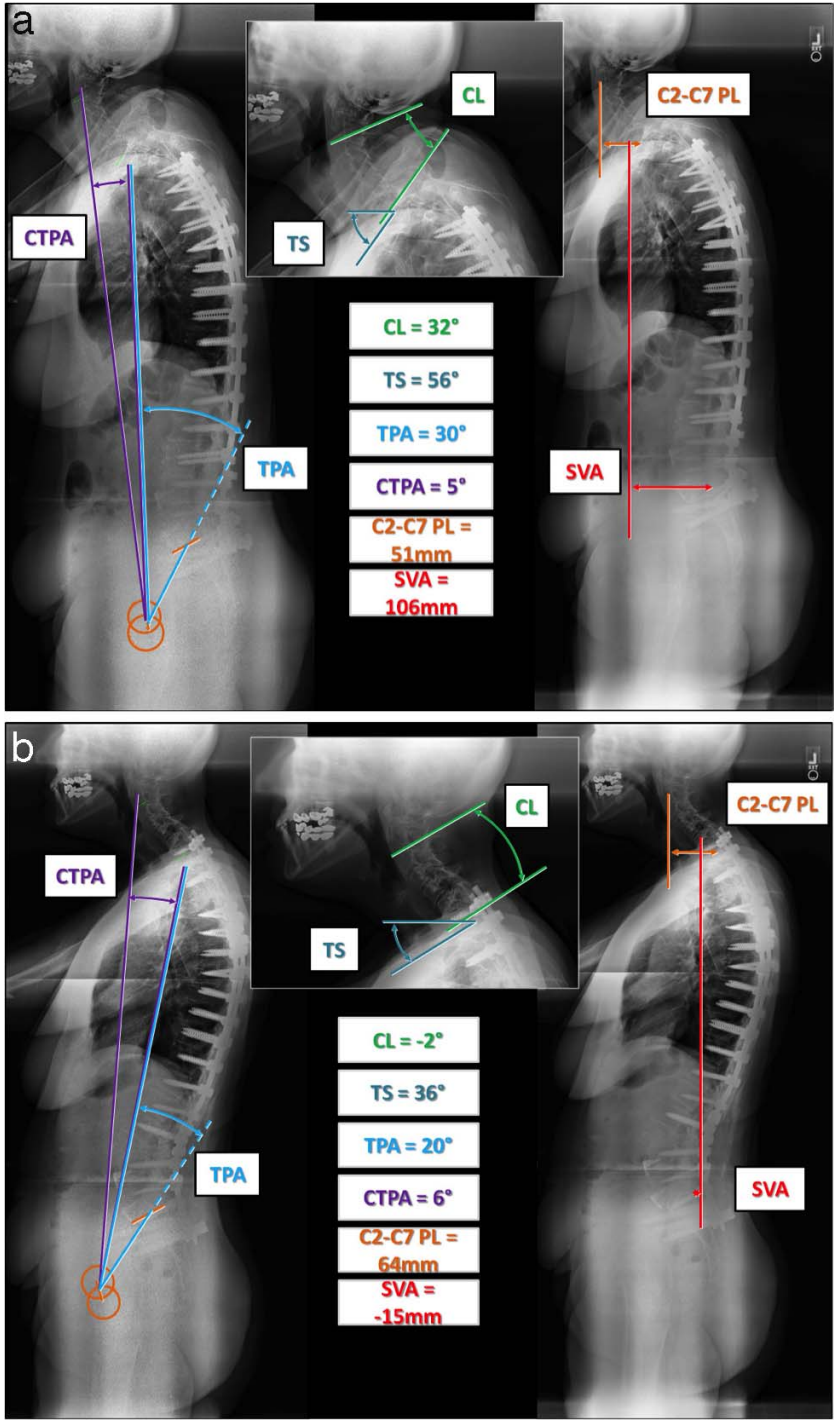

Figure 3. (a) Preoperative x-rays of a patient with thoracolumbar deformity from the uncompensated cervical sagittal deformity (CSD) group. Though there is lordotic alignment of the cervical spine, there is a mismatch in T1 slope and cervical lordosis $\left(\mathrm{TS}-\mathrm{CL}>17^{\circ}\right.$ ). Cervical plumb line, TS-CL, and CTPA are elevated. (b) Postoperative x-rays of the same patient from the uncompensated group. There has been good global correction, but there has been progression of the cervical deformity with kyphotic alignment of the cervical spine, an increase in the cervical plumb line, a progression in the mismatch of T1 slope and cervical lordosis, and an increase in the cervico-thoracic pelvic angle. Abbreviations: CL, cervical lordosis; TS, T1 slope; TPA, T1 pelvic angle; CTPA, cervico-thoracic pelvic angle; C2-C7 PL, cervical plumb line; SVA, sagittal vertical axis.

\section{RESULTS}

\section{Study Sample}

A total of 223 consecutive patients who received thoracolumbar pedicle subtraction osteotomy met inclusion criteria. Of the patients $70 \%$ were women and $30 \%$ were men, with mean age 57.56 years (range, 20 to 81 years, $\mathrm{SD}=12.9$ years) and average body mass index $27.79 \mathrm{~kg} / \mathrm{m}^{2}$ (range, 17.6 to 62.8 $\mathrm{kg} / \mathrm{m}^{2}, \mathrm{SD}=6.97 \mathrm{~kg} / \mathrm{m}^{2}$ ). The majority of the patients $(\mathrm{n}=170,76.2 \%)$ had a history of prior spine surgery: $81.2 \%$ had lumbar osteotomy and $18.8 \%$ had thoracic osteotomy. The most common osteotomy sites were L3 (34.1\%) and L4 (21.1\%).

The analysis of the preoperative radiographic parameters by Scoliosis Research Society-Schwab classification revealed that $60.5 \%$ of patients had marked global malalignment (SVA $>9.5 \mathrm{~mm})$, $64.1 \%$ had marked PI-LL mismatch (PI-LL > $20^{\circ}$ ), and $49.3 \%$ had marked pelvic tilt $\left(>30^{\circ}\right)$. There was a significant coronal deformity (ie, coronal $\mathrm{Cobb}>30^{\circ}$ ) in $51 \%$ of patients, consisting of $9 \%$ with thoracic curvature greater than $30^{\circ}, 12 \%$ with double major curves, and 30\% with thoracolumbar curves. Postoperatively, $11.7 \%$ had marked SVA, $13 \%$ of patients had marked PI-LL, and $25.1 \%$ had marked pelvic tilt. As illustrated in Table 1 , the comparison of preoperative and postoperative radiographic parameters revealed significant changes, with the exception of the CPL and TS.

\section{Radiographic Correlations}

CTPA correlated with CPL as an angular analog to this linear measure of cervical alignment with preoperative $r=.85$, and postoperative $r=.699$, (all $P<.001$; Figure 5). C2 pelvic angle correlated with C2-S1 plumb line with preoperative $r=.756$ and postoperative $r=.683$ (all $P<.001$ ). TS-CL correlated with CTPA with preoperative $r=.52$, and postoperative $r=.37$, and TS-CL correlated with CPL with preoperative $r=.57$ and postoperative $r=.37$ (all $P<.001$ ).

\section{Threshold of Cervical Deformity}

One-year postoperative values were used in a linear regression analysis to determine the corresponding values of TS-CL for the known pathologic value of $\mathrm{CPL}=4 \mathrm{~cm}^{7}$ TS-CL was found to significantly correlate with CPL and led to the following equation $\left(P=.0001, r=.479, r^{2}=.230\right)$ : $\mathrm{TS}-\mathrm{CL}=4.749+0.306 \times \mathrm{CPL}$. A TS-CL of $17^{\circ}$ was found to correspond to a CPL of $4 \mathrm{~cm}$.

\section{Comparison of Compensated Versus Uncompensated Alignment Groups}

The stratification by group identified 123 patients in the compensated group and 100 patients in the uncompensated group, without any significant difference in demographic parameters (Table 2). There were no significant differences between the 



Figure 4. (a) Preoperative $\mathrm{x}$-rays of a thoracolumbar deformity patient from the compensated group (no cervical deformity). The magnitude of the cervical lordosis matches that of the T1 slope (TS-CL $<17^{\circ}$ ), demonstrating that there is good cervical compensation for the large global deformity allowing for horizontal gaze. (b) Postoperative x-rays of the same patient from the compensated group. Good global correction has been achieved and reciprocal change has occurred, with a decrease in the cervical sagittal alignment. The magnitude of the cervical lordosis remains in balance with the T1 slope. Abbreviations: CL, cervical lordosis; TS, T1 slope; TPA, T1 pelvic angle; CTPA, cervico-thoracic pelvic angle; C2-C7 PL, cervical plumb line; SVA, sagittal vertical axis.

two groups in any of the following sagittal alignment parameters: thoracic kyphosis, lumbar lordosis, pelvic tilt, PI-LL, and global measures including the SVA, T1 pelvic angle, and C2 pelvic angle.

The comparison of preoperative radiographic features (Table 3 ) revealed that the uncompensated group had greater CPL (4.5 versus 3.4; $P<.001$ ), a greater $\mathrm{C} 2$ slope $\left(21.4^{\circ}\right.$ versus $\left.9.13^{\circ}\right)$, a smaller cervical lordosis $\left(12.5^{\circ}\right.$ versus $\left.23^{\circ}\right)$, a greater mismatch between TS and CL (TS-CL: $22.2^{\circ}$ versus $\left.10.2^{\circ}\right)$, and a greater CTPA $\left(3.4^{\circ}\right.$ versus $\left.2.3^{\circ}\right)$.
Whereas these significant differences were maintained postoperatively, the compensated cohort of patients had a decrease in TS-CL (from $10.2^{\circ}$ to $8.0^{\circ}$ ) with SVA correction, the uncompensated patients had an increase in TS-CL (from $22.3^{\circ}$ to $26.8^{\circ}$ ), with all $P<.001$.

The analysis of correlations within each group demonstrated that reciprocal change in cervical alignment occurred in the compensated group as CL decreased with SVA correction $(r=.39, P<$ $.001)$, but there was no such correlation in the uncompensated group $(r=.08, P=.435)$.

Table 1. Comparison of the preoperative and postoperative radiographic parameters following three-column osteotomy (paired $t$ test).

\begin{tabular}{|c|c|c|c|c|c|}
\hline & \multicolumn{2}{|c|}{ Preoperative } & \multicolumn{2}{|c|}{1 Year } & \multirow[b]{2}{*}{ Paired $t$ test; $P$ Value } \\
\hline & Mean & SD & Mean & SD & \\
\hline CPL & 38.74 & 19.91 & 38.32 & 19.17 & .684 \\
\hline $\mathrm{C} 2 \mathrm{~S}$ & 14.62 & 12.99 & 16.46 & 12.77 & .019 \\
\hline CL & 18.31 & 17.49 & 11.61 & 16.63 & .0001 \\
\hline T1S & 33.89 & 15.17 & 28.08 & 13.18 & .266 \\
\hline TS-CL & 15.58 & 12.89 & 16.46 & 12.22 & .0001 \\
\hline TK_T2 & -36.99 & 25.26 & -46.68 & 16.64 & .0001 \\
\hline $\mathrm{TK}_{-}^{-} \mathrm{T} 4$ & -34.40 & 23.77 & -39.77 & 15.71 & .0001 \\
\hline $\mathrm{LL}^{-}$ & 30.94 & 25.20 & 54.91 & 15.07 & .0001 \\
\hline PT & 30.32 & 12.97 & 22.85 & 11.45 & .0001 \\
\hline PI-LL & 26.89 & 26.56 & 3.25 & 16.75 & .0001 \\
\hline SVA & 117.46 & 81.65 & 29.73 & 57.28 & .0001 \\
\hline CTPA & 2.78 & 2.09 & 3.55 & 1.92 & .0001 \\
\hline CPA & 35.28 & 15.50 & 22.06 & 12.25 & .0001 \\
\hline TPA & 32.50 & 15.94 & 18.51 & 12.01 & .0001 \\
\hline
\end{tabular}

Abbreviations: CPL, C2-C7 plumb line; C2S, C2 slope; CL, cervical lordosis; T1S, T1 slope; TS-CL, T1 slope minus CL; TK T2, T2 T12 kyphosis; TK T4, T4_T12 kyphosis; LL, lumbar lordosis; PT, pelvic tilt; PI-LL, pelvic incidence minus LL; SVA, sagittal vertical axis; CTPA, cervico-thoracic pelvic angle; CPA, C 2 pelvic angle; TPA, T1 pelvic angle. 


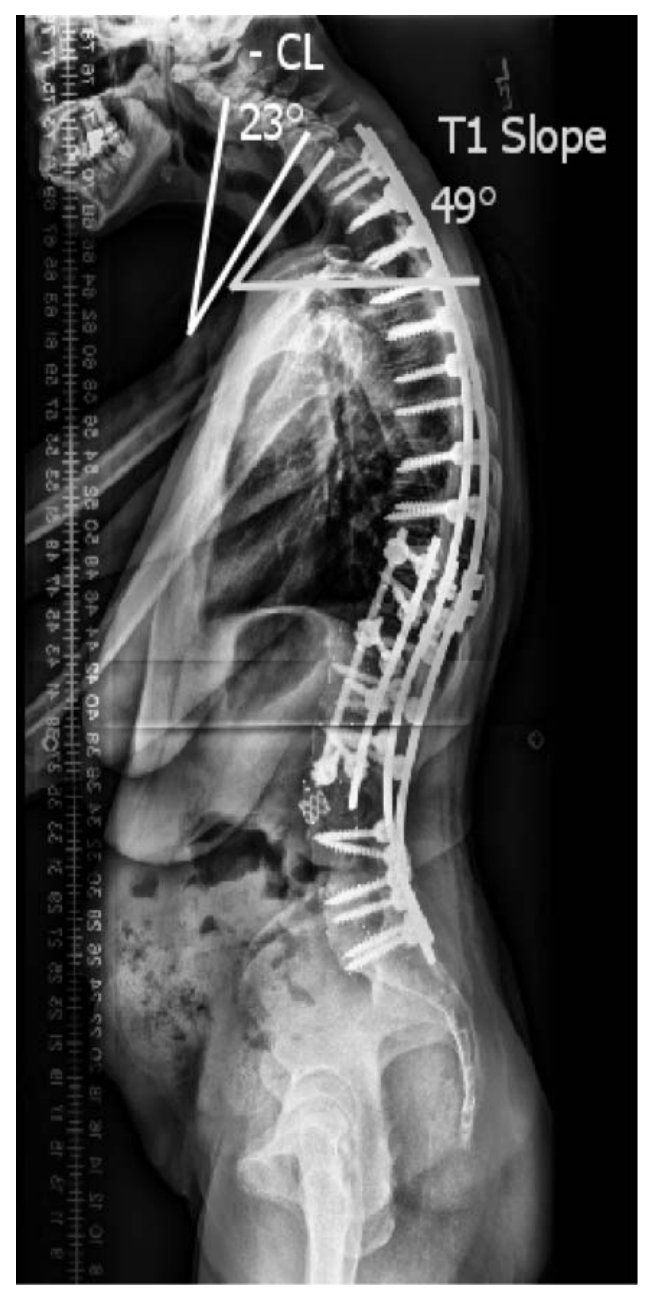

Figure 5. Patient with thoracolumbar deformity after pedicle subtraction osteotomy with a long fusion to the pelvis; patient developed cervical sagittal deformity. T1 slope minus cervical lordosis (TS-CL) is $72^{\circ}$.

One-year postoperative values were used in a linear regression analysis to determine the corresponding values of TS-CL for the known pathologic value of $\mathrm{CPL}=4 \mathrm{~cm}^{7}$ TS-CL was found to significantly correlate with CPL and led to the following equation $\left(P=.0001, r=.479, r^{2}=.230\right)$ : $\mathrm{TS}-\mathrm{CL}=4.749+0.306 \times \mathrm{CPL}$. A TS-CL of $17^{\circ}$ was found to correspond to a CPL of $4 \mathrm{~cm}$.

\section{Comparison of TS-CL With Established Parameters, CPL and CK, as Determinants of CSD}

Of the 100 patients in the uncompensated group with a TS-CL $>17^{\circ}, 36$ did not meet deformity criteria by established measures $(\mathrm{CPL}>4 \mathrm{~cm}$, or $\mathrm{CL}<0^{\circ}$ ). Among this group of 36 patients, the mean preoperative CPL was $29.07 \pm 9.01 \mathrm{~mm}$ and the preoperative $\mathrm{CL}$ was $15.36^{\circ} \pm 11.33^{\circ}$. Postoperatively both alignment parameters worsened with
Table 2. Comparison of demographic parameters between the compensated and the uncompensated groups.

\begin{tabular}{lccc}
\hline & $\begin{array}{c}\text { Compensated } \\
(\mathbf{n}=\mathbf{1 2 3})\end{array}$ & $\begin{array}{c}\text { Uncompensated } \\
(\mathbf{n}=\mathbf{1 0 0})\end{array}$ & $\boldsymbol{P}$ \\
\hline Age, y & 57.7 & 57.4 & NS \\
Female, \% & 70.7 & 76.3 & NS \\
Male, \% & 29.3 & 13.7 & .05 \\
Previous surgery, \% & 74.8 & 78 & NS \\
\hline
\end{tabular}

Abbreviation: NS, not significant.

a mean CPL of $36.61 \pm 16.7 \mathrm{~mm}$ and a mean CL of $1.03^{\circ} \pm 12.6^{\circ}$.

Of the 123 patients in the compensated group with a TS-Cl $<17^{\circ}$, only 2 had deformities by the established criteria $\left(\mathrm{CPL}>4 \mathrm{~cm}\right.$, or $\mathrm{CL}<0^{\circ}$ ). Among these 2 patients, the mean CPL improved from a preoperative value of $65.22 .07 \pm 22.88 \mathrm{~mm}$ to a postoperative value of $47.14 \pm 13.8 \mathrm{~mm}$ and the mean preoperative $\mathrm{CL}$ of $-3.35^{\circ} \pm 1.62^{\circ}$ improved to a postoperative value of $15.2^{\circ} \pm 8.9^{\circ}$.

\section{Predictors of TS-CL Over $17^{\circ}$}

Logistic regression modeling was used to determine predictors of postoperative CSD (TS-CL over $17^{\circ}$ ). The results of the logistic regression model are presented in Table 3. Positive predictors of postoperative CSD (predictors associated with higher odds of having a postoperative TS-CL over $17^{\circ}$ ) include baseline TS-CL $>17^{\circ}$ (odds ratio [OR], 2.78; $P=$ .007), longer fusion (OR, 1.3 per increase of 3 segments of fusion; $P=.033$ ), and baseline CTPA (OR, 1.24 per degree; $P=.029$ ). Having a preoperative coronal imbalance showed a trend toward decreasing the odds of postoperative CSD (OR, 0.59; $P=.1$; Table 4).

\section{DISCUSSION}

Sagittal spinal alignment has been well established as a primary driver of disability in patients with thoracolumbar deformity. ${ }^{20,21}$ It is becoming increasingly more apparent that cervical sagittal alignment is similarly an important determinant of health-related quality of life measures. ${ }^{7,22,23}$ Tang et $\mathrm{al}^{7}$ studied 113 patients undergoing posterior cervical fusion, and positive cervical sagittal balance was associated with greater disability by validated outcome measures including the neck disability index and 36-item short form health survey. Villavicencio et $\mathrm{al}^{22}$ demonstrated that if cervical sagittal alignment was maintained or improved, SF36 and NDI outcomes were improved among 122 
Table 3. Comparison of radiographic features (pre, post, and change) between the compensated (C) and uncompensated (U) groups.

\begin{tabular}{|c|c|c|c|c|c|c|}
\hline & \multicolumn{2}{|c|}{ Preoperative } & \multicolumn{2}{|c|}{1 Year } & \multicolumn{2}{|c|}{ Change at 1 Year } \\
\hline & $\mathrm{C}$ & $\mathbf{U}$ & $\mathrm{C}$ & $\mathbf{U}$ & $\mathrm{C}$ & $\mathbf{U}$ \\
\hline CPL & $33.76^{*}$ & $44.87 *$ & $31.57 *$ & $46.63 *$ & -2.18 & 1.76 \\
\hline $\mathrm{C} 2 \mathrm{~S}$ & $9.13^{*}$ & $21.36^{*}$ & $7.58 *$ & $27.39 *$ & $-1.55^{*}$ & $6.03^{*}$ \\
\hline CL & $23.02 *$ & $12.53^{*}$ & $18.91 *$ & $2.64 *$ & -4.10 & -9.89 \\
\hline T1S & 33.25 & 34.69 & 26.91 & 29.51 & -6.34 & -5.17 \\
\hline TS-CL & $10.23 *$ & $22.16^{*}$ & $8.00 *$ & $26.87^{*}$ & $-2.23^{*}$ & $4.72 *$ \\
\hline TK T2 & -36.27 & -37.88 & -46.80 & -46.53 & -10.53 & -8.66 \\
\hline TK_- T4 & -33.96 & -34.94 & -40.82 & -38.50 & -6.85 & -3.56 \\
\hline $\mathrm{LL}^{-}$ & 29.77 & 32.38 & 56.02 & 53.54 & 26.25 & 21.16 \\
\hline PT & 30.19 & 30.47 & 21.68 & 24.30 & -8.51 & -6.17 \\
\hline PI-LL & 27.30 & 26.40 & 1.46 & 5.44 & -25.83 & -20.96 \\
\hline SVA & 119.90 & 114.45 & 29.24 & 30.34 & -90.65 & -84.12 \\
\hline CTPA & $2.29 *$ & $3.38^{*}$ & $2.93 *$ & $4.31 *$ & 0.65 & 0.93 \\
\hline CPA & 35.01 & 35.60 & 20.50 & 23.97 & -14.51 & -11.63 \\
\hline TPA & 32.73 & 32.22 & 17.57 & 19.66 & -15.16 & -12.55 \\
\hline
\end{tabular}

Abbreviations: CPL, C2-C7 plumb line; C2S, C2 slope; CL, cervical lordosis; T1S, T1 slope; TS-CL, T1 slope minus CL; TK T2, T2 T12 kyphosis; TK T4, T4_T12 kyphosis; LL, lumbar lordosis; PT, pelvic tilt; PI-LL, pelvic incidence minus LL; SVA, sagittal vertical axis; CTPA, cervico-thoracic pelvic angle; CPA, C2 pelvic angle; TPA, T1 pelvic angle.

* Indicates significant difference between values.

patients undergoing anterior cervical discectomy and fusion.

However, CSD is difficult to define, especially when there is concurrent thoracolumbar deformity. Several authors have noted that an underlying thoracolumbar sagittal malalignment causes positive cervical sagittal balance. ${ }^{1,23-26}$ This reciprocal cervical malalignment reverses with correction of the underlying thoracolumbar deformity. Therefore, a positive cervical sagittal alignment in the setting of thoracolumbar sagittal malalignment is not necessarily indicative of primary cervical deformity.

Likewise, cervical kyphosis is considered to be a pathologic indicator of cervical deformity. However, many have noted that cervical kyphosis in a standing individual can be a flexible physiologic state that depends on global standing balance and the underlying spinopelvic morphology. ${ }^{10,25}$ The presence of cervical kyphosis is, therefore, not necessarily indicative of a primary cervical deformity.

We used the relationship between TS and CL to determine patients who have concurrent primary CSD and thoracolumbar deformity. The balance between pelvic incidence and lumbar lordosis has

Table 4. Predictors of postoperative cervical sagittal deformity using logistic regression.

\begin{tabular}{lccc}
\hline & Odds Ratio & SE & $\boldsymbol{P}$ Value \\
\hline Baseline TS-CL > 17 & 2.79 & 1.06 & .007 \\
Coronal imbalance & 0.59 & 0.19 & .1 \\
Number of levels fused & 1.29 & 0.15 & .03 \\
Baseline CTPA & 1.24 & 0.12 & .029 \\
Cervical lordosis & 0.97 & 0.01 & .004 \\
\hline
\end{tabular}

Abbreviations: TS-CL, T1 slope minus CL; CTPA, cervico-thoracic pelvic angle. been shown to be an important relationship in the determination of lumbar flatback deformity. ${ }^{11-13,26}$ Similarly, TS-CL is a relationship that can define primary CSD (Figure 1). ${ }^{8,14,15}$ The relationship between TS and CL is analogous to that of SS and LL. SS has been shown to be highly correlated to $\mathrm{LL}$ in asymptomatic patients. ${ }^{2,14}$ In fact the correlation between SS and LL was stronger than that for pelvic incidence and LL. ${ }^{14}$

In a retrospective study, Hyun et $\mathrm{al}^{27}$ showed that there is a significant positive correlation between C2-C7 and SVA and TS-CL, with the latter measurement significantly affecting cervical alignment. The study revealed that a mismatch greater than $26.1^{\circ}$ corresponded to positive cervical sagittal malalignment, defined as C2-C7 SVA greater than $50 \mathrm{~mm}^{27}$

In our study, we used a linear regression analysis to determine the postoperative TS-CL that corresponds to a CPL of $4 \mathrm{~cm}$. Tang et $\mathrm{al}^{7}$ previously demonstrated that a CPL of greater than $4 \mathrm{~cm}$ is associated with poor health measures in patients with primary cervical deformity. We correlated the postoperative values of TS-CL and CPL because correction of the underlying thoracolumbar deformities in our patient population should lead to a reciprocal correction of any cervical malalignment. Following the thoracolumbar deformity correction, any residual positive cervical sagittal alignment is more likely to be the result of an underlying primary cervical deformity. By determining that a TS-CL threshold of $17^{\circ}$ correlates to a CPL of $4 \mathrm{~cm}$, we used this threshold to categorize the patients into the compensated and uncompensated groups. 
We found it interesting that the compensated group (TS-CL $<17^{\circ}$ ) had improved cervical alignment following correction of the underlying thoracolumbar deformities. Conversely, the postoperative alignment of the uncompensated cohort (TS$\mathrm{CL}>17^{\circ}$ ) did not demonstrate any reciprocal change in CL and there was a paradoxical increase in the CPL and an increase in the cervico-thoracic pelvic angle (CTPA), which is a novel global measure of cervical sagittal alignment (Figures 2 through 5$).^{28}$

The TS-CL measure identified 36 patients with $\mathrm{CSD}\left(\mathrm{TS}-\mathrm{CL}>17^{\circ}\right.$ ) that did not meet criteria for deformity by established measures (CPL $>4 \mathrm{~cm}$, or $\mathrm{CL}<0^{\circ}$ ). In these 36 patients, the mean $\mathrm{CPL}$ worsened preoperatively to postoperatively (from $29.07 \pm 9.01 \mathrm{~mm}$ to $36.61 \pm 16.7 \mathrm{~mm}$ ) and the mean CL worsened from $15.36^{\circ} \pm 11.33^{\circ}$ to $1.03^{\circ} \pm$ $12.6^{\circ}$. Conversely, there were only 2 patients from the compensated group with deformities by the established criteria. In both patients the CPL and CL improved preoperatively to postoperatively. This suggests that the TS-CL measure can be useful in defining CSD and in predicting those patients who may have a tendency to worsen in postoperative alignment.

A linear regression analysis was used to determine risk factors for the development of cervical sagittal malalignment (TS-CL above threshold). Potential predictors were identified using univariate analysis, and a statistical model was built using a combination of backward elimination and bootstrap selection. The model fit was assessed using the Hosmer-Lemeshow test and the $\mathrm{C}$ statistic/receiver operating characteristic curve; internal validation of the model was performed using 10-fold crossvalidation. Positive predictors of postoperative CSD included baseline TS-CL $>17^{\circ}(\mathrm{OR}, 2.78 ; P$ $=.007)$, longer fusion (OR, $1.3 ; P=.033)$, and baseline CTPA (OR, 1.24 per degree; $P=.029$ ). This suggests that patients with preoperative cervical deformities, as defined by a larger baseline TS-CL mismatch and high CTPA, were more likely to maintain postoperative cervical deformities and unlikely to undergo reciprocal change of cervical alignment. Moreover, this analysis shows that those with longer fusions were more likely to have postoperative cervical deformities. This may be due to diminished capacity for adjacent cervical regional compensation or the result of disruption of the cervical paraspinal muscle attachments and the capsuloligamentous complex at the cervicothoracic junction during exposure and instrumentation.

A primary limitation of the study is that this data set did not include health-related quality of life measures and so the threshold of CSD (CPL > 4 $\mathrm{cm})$ had to be extrapolated from the literature. ${ }^{6}$ However, it is important to note that a questionnaire specific to cervical sagittal alignment does not exist. Another limitation of this study is the retrospective design, although the analyses were based on prospectively collected data. The strengths of the study include the contribution of cases from multiple spinal-deformity centers and the use of multiple standardized measures of regional and global sagittal spinal alignment.

This study demonstrates the utility of TS-CL to determine when primary CSD is present concurrently with thoracolumbar deformity. Further research is needed to establish the balance between TS and CL (TS-CL) as an integral CSD parameter, correlated with health-related quality of life measures.

\section{REFERENCES}

1. Blondel BS, Schwab F, Ames CP, et al. The crucial role of cervical alignment in regulating sagittal spino-pelvic alignment in human standing posture. Podium presented at 19th International Meeting on Advanced Spine Techniques; July 1821, 2012; Istanbul, Turkey.

2. Berthonnaud E, Dimnet J, Roussouly P, Labelle H. Analysis of the sagittal balance of the spine and pelvis using shape and orientation parameters. J Spinal Disord Tech. 2005;18(1):40-47.

3. Ha Y, Schwab F, Lafage V, et al. Reciprocal changes in cervical spine alignment after corrective thoracolumbar deformity surgery. Eur Spine J. 2014;23(3):552-559. https://doi.org/ 10.1007/s00586-013-2953-8.

4. Klineberg E, Schwab F, Ames C, et al. Acute reciprocal changes distant from the site of spinal osteotomies affect global postoperative alignment. Adv Orthop. 2011;2011:415946.

5. Lafage V, Schwab F, Skalli W, et al. Standing balance and sagittal plane spinal deformity: analysis of spinopelvic and gravity line parameters. Spine (Phila Pa 1976). 2008;33(14):15721578.

6. Smith J, Shaffrey, C, Lafage, V, et al. Prevalence and type of cervical deformity among 470 adults with thoracolumbar deformity. Presented at the 48th Annual Meeting and Course of the Scoliosis Research Society; September 18-21, 2013; Lyon, France.

7. Tang JA, Scheer JK, Smith JS, et al. The impact of standing regional cervical sagittal alignment on outcomes in posterior cervical fusion surgery. Neurosurgery. 2012;71(3):662669, discussion 669.

8. Smith JS, Shaffrey CI, Lafage V, et al. Spontaneous improvement of cervical alignment after correction of global 
sagittal balance following pedicle subtraction osteotomy. $J$ Neurosurg Spine. 2012;17(4):300-307.

9. Kuntz CT, Levin LS, Ondra SL, Shaffrey CI, Morgan CJ. Neutral upright sagittal spinal alignment from the occiput to the pelvis in asymptomatic adults: a review and resynthesis of the literature. J Neurosurg Spine. 2007;6(2):104-112.

10. Gore DR, Sepic SB, Gardner GM. Roentgenographic findings of the cervical spine in asymptomatic people. Spine. 1986;11(6):521-524.

11. Roussouly P, Gollogly S, Berthonnaud E, Dimnet J. Classification of the normal variation in the sagittal alignment of the human lumbar spine and pelvis in the standing position. Spine. 2005;30(3):346-353.

12. Vaz G, Roussouly P, Berthonnaud E, Dimnet J. Sagittal morphology and equilibrium of pelvis and spine. Eur Spine J. 2002;11(1):80-87.

13. Schwab F, Lafage V, Patel A, Farcy JP. Sagittal plane considerations and the pelvis in the adult patient. Spine (Phila Pa 1976). 2009;34(17):1828-1833.

14. Vialle R, Levassor N, Rillardon L, Templier A, Skalli W, Guigui P. Radiographic analysis of the sagittal alignment and balance of the spine in asymptomatic subjects. $J$ Bone Joint Surg Am. 2005;87(2):260-267.

15. Kim TH, Lee SY, Kim YC, Park MS, Kim SW. T1 slope as a predictor of kyphotic alignment change after laminoplasty in patients with cervical myelopathy. Spine (Phila Pa 1976). 2013;38(16):E992-E997.

16. Schwab FB, Blondel B, Chay E, et al. The comprehensive anatomical spinal osteotomy classification. Neurosurgery. 2014;74(1):112-120.

17. Rillardon L, Levassor N, Guigui P, et al. Validation of a tool to measure pelvic and spinal parameters of sagittal balance [in French]. Rev Chir Orthop Reparatrice Appar Mot. 2003;89(3):218-227.

18. El Fegoun AB, Schwab F, Gamez L, Champain N, Skalli W, Farcy JP. Center of gravity and radiographic posture analysis: a preliminary review of adult volunteers and adult patients affected by scoliosis. Spine (Phila Pa 1976). 2005;30(13):1535-1540.

19. Schwab F, Ungar B, Blondel B, et al. Scoliosis Research Society-Schwab adult spinal deformity classification: a validation study. Spine (Phila Pa 1976). 2012;37(12):10771082.

20. Schwab FJ, Blondel B, Bess S, et al. Radiographical spinopelvic parameters and disability in the setting of adult spinal deformity: a prospective multicenter analysis. Spine. 2013;38(13):E803-E812.

21. Smith JS, Klineberg E, Schwab F, et al. Change in classification grade by the SRS-Schwab adult spinal deformity classification predicts impact on health-related quality of life measures: prospective analysis of operative and non-operative treatment. Spine (Phila Pa 1976) 2013;38(19);1663-1671.

22. Villavicencio AT, Babuska JM, Ashton A, et al. Prospective, randomized, double-blind clinical study evaluating the correlation of clinical outcomes and cervical sagittal alignment. Neurosurgery. 2011;68(5):1309-1316, discussion 1316.

23. Scheer JK, Tang JA, Smith JS, et al. Cervical spine alignment, sagittal deformity, and clinical implications. $J$ Neurosurg Spine. 2013;19(2):141-159.

24. Park MS, Moon SH, Lee HM, et al. The effect of age on cervical sagittal alignment: normative data on 100 asymptomatic subjects. Spine. 2013;38(8):E458-E463.

25. Kuntz Ct, Shaffrey CI, Ondra SL, et al. Spinal deformity: a new classification derived from neutral upright spinal alignment measurements in asymptomatic juvenile, adolescent, adult, and geriatric individuals. Neurosurgery. 2008;63(3 suppl):25-39.

26. Farcy JP, Schwab FJ. Management of flatback and related kyphotic decompensation syndromes. Spine (Phila $\mathrm{Pa}$ 1976). 1997;22(20):2452-2457.

27. Hyun S-J, Kim K-J, Jahng T-A, Kim H-J. Relationship between $\mathrm{T} 1$ slope and cervical alignment following multilevel posterior cervical fusion surgery. Spine. 2016;41(7):E396-E402.

28. Themistocles S, Protopsaltis MNB, Terran JS, et al. Cervical sagittal deformity develops after PJK in adult thoracolumbar deformity correction: radiographic analysis utilizing a novel global sagittal parameter, the CTPA. Presented at the 20th International Meeting on Advanced Spine Techniques; July 10-13, 2013; Vancouver, British Columbia, Canada.

Corresponding Author: Themistocles Protopsaltis, MD, New York University Langone Medical Center, Hospital for Joint Diseases, Department of Orthopaedic Surgery, 301 East 17th Street, New York, NY 10003. Phone: (646) 501-7200; Fax: (212) 598-7654.

Published 15 August 2018

This manuscript is generously published free of charge by ISASS, the International Society for the Advancement of Spine Surgery. Copyright $@ 2018$ ISASS. To see more or order reprints or permissions, see http://ijssurgery.com. 